\title{
3D pelvimetry and biometric measurements: a surgical perspective for colorectal resections
}

\author{
Laura Lorenzon ${ }^{1,2}$ (D) $\cdot$ Fabiano Bini ${ }^{3} \cdot$ Federica Landolfi $^{2} \cdot$ Serena Quinzi ${ }^{3} \cdot$ Genoveffa Balducci $^{2} \cdot$ Franco Marinozzi $^{3}$. \\ Alberto Biondi ${ }^{1} \cdot$ Roberto Persiani $^{1} \cdot$ Domenico D'Ugo $^{1} \cdot$ Flavio Tirelli $^{1} \cdot$ Elsa lannicelli ${ }^{2}$
}

Accepted: 11 November 2020 / Published online: 23 November 2020

(C) The Author(s) 2020

\begin{abstract}
Purpose Male sex, high BMI, narrow pelvis, and bulky mesorectum were acknowledged as clinical variables correlated with a difficult pelvic dissection in colorectal surgery. This paper aimed at comparing pelvic biometric measurements in female and male patients and at providing a perspective on how pelvimetry segmentation may help in visualizing mesorectal distribution. Methods A 3D software was used for segmentation of DICOM data of consecutive patients aged 60 years, who underwent elective abdominal CT scan. The following measurements were estimated: pelvic inlet, outlet, and depth; pubic tubercle height; distances from the promontory to the coccyx and to $\mathrm{S} 3 / \mathrm{S} 4$; distance from $\mathrm{S} 3 / \mathrm{S} 4$ to coccyx's tip; ischial spines distance; pelvic tilt; offset angle; pelvic inlet angle; angle between the inlet/sacral promontory/coccyx; angle between the promontory/coccyx/pelvic outlet; S3 angle; and pelvic inlet to pelvic depth ratio. The measurements were compared in males and females using statistical analyses.

Results Two-hundred patients (M/F 1:1) were analyzed. Out of 21 pelvimetry measurements, 19 of them documented a significant mean difference between groups. Specifically, female patients had a significantly wider pelvic inlet and outlet but a shorter pelvic depth, and promontory/sacral/coccyx distances, resulting in an augmented inlet/depth ratio when comparing with males $(p<0.0001)$. The sole exceptions were the straight conjugate $(p=0.06)$ and $\mathrm{S} 3$ angle $(p=0.17)$. 3D segmentation provided a perspective of the mesorectum distribution according to the pelvic shape.

Conclusion Significant differences in the structure of pelvis exist in males and females. Surgeons must be aware of the pelvic shape when approaching the rectum.
\end{abstract}

Keywords Rectal cancer $\cdot$ TaTME $\cdot$ Total mesorectal excision $\cdot$ 3D imaging

\section{Introduction}

Over the last few years, the surgical treatment of rectal cancer evolved at a rapid pace. Implementation of technologies led to the introduction of mini-invasive techniques, but their

Laura Lorenzon and Fabiano Bini contributed equally to this work.

Laura Lorenzon

laura.lorenzon@policlinicogemelli.it

1 General Surgery Unit, Fondazione Policlinico Universitario Agostino Gemelli IRCCS, Catholic University, Rome, Italy

2 Surgical and Medical Department of Translational Medicine, Sant'Andrea Hospital, Faculty of Medicine and Psychology, "Sapienza" University of Rome, 00185 Rome, RM, Italy

3 Department of Mechanical and Aerospace Engineering, "Sapienza" University of Rome, via Eudossiana 18, 00184 Rome, Italy widespread clinical adoption was impaired due to several reasons, including costs (i.e. robotics procedure), and technical difficulties (i.e. limitation of the surgical instruments and articulation of the laparoscopic devices) [1].

If availability, limitations, and costs of surgical devices could represent issues, also patients' and tumour characteristics influenced surgical choices. Male sex, high BMI, narrow pelvis, and bulky mesorectum were all acknowledged in literature as the clinical variables correlated with a difficult pelvic dissection and incomplete mesorectal excision or positive distal/radial margins [2].

Nevertheless, a novel approach is currently emerging which combines the benefits of mini-invasive surgery with the principles of surgical oncology and total mesorectal excision (TME). Trans-anal TME (TaTME) procedures gained interest in relation to a reduced conversion rate and longer distal resection margins comparing laparoscopic trans- 
abdominal low rectal resections [3]. Along with its benefits, few pitfalls started to emerge when approaching the rectum bottom-up, since the surgical field is demanding and performing a mesorectal dissection could be difficult in relation to the challenging anatomy $[4,5]$. New adverse events such as nerve, vessel, and urethral injuries have been described and several authors reported new tips for dissection and preservation of the correct planes $[4,6]$. Of note, up to $40 \%$ of current TaTME literature focuses on its complications (Supplement Figure 1).

Though the notion that the surgical procedure is easier in a wider pelvis is a well-recognized matter among colorectal surgeons, the anatomy and its biometric measurements are gaining interest; indeed, a complete understanding of the pelvis anatomy is essential for colorectal surgeons and it can provide the basis for a sharp dissection with curative intent during the resection of rectal cancers [7].

Pelvimetry, measurement of pelvic bony dimensions, has been performed for over half a century, in attempts to predict cephalo-pelvic disproportion prior to labour. $2 \mathrm{D}$ and $3 \mathrm{D}$ pelvimetry reconstructions, both using CT scans or MRI [8, 9], are gaining a momentum in this field, with the main outcomes of predicting surgical difficulties, or achieving a complete mesorectal plane, negative circumferential margins, or a sphincter-saving procedure [9-11].

Pelvimetry has been also applied to modern radiology to investigate differences in pelvic diameters of female and male patients undergoing rectal surgery procedures, even if with contradicting results $[8,12]$.

The aim of this study was to investigate differences between female and male patients of the pelvic biometric measurements obtained using 3D segmentation of CT scans and to provide a perspective on how pelvimetry may help in visualizing mesorectum distribution.

\section{Materials and methods}

All consecutive patients born in 1955, 1956, and 1957 who underwent an elective or emergency abdominal CT scan with or without contrast enhancement at Sant'Andrea Hospital of Rome respectively during 2015, 2016, and 2017, independently from the indication, were considered eligible and retrospectively reviewed. The choice of 60 -year-old patients was made consistently with the aim of this study which had the objective to evaluate pelvimetry in a typical adult, asymptomatic, and at average risk population for colorectal cancer [13]. Patients who had pelvic and/or femur fractures, those with fixation devices, or those with incomplete pelvic bone acquisitions were excluded. Of note, the reason to include emergencies and elective procedures with or without contrast was motivated by the fact that soft tissues were not the objective of $3 \mathrm{D}$ segmentation, and thus, exclusion criteria were limited to those conditions impairing imaging modelling.

Computed tomography scans were performed with multidetector CT equipment, GE Light Speed 16. The following technical parameters were used: $120 \mathrm{kV} ; 120$ to $180 \mathrm{~mA}$; gantry rotation time, $0.5 \mathrm{~s}$; beam collimation, $16 \times 1.25 \mathrm{~mm}$; beam pitch, 1; and reconstruction thickness, $2.5 \mathrm{~mm}$.

Pelvimetry DICOM data from selected patients were analyzed using 3D Slicers Software, version 4.5.0-1. From the axial plane view, each slice was processed by means of automatic segmentation algorithm level tracing effect, available in the Editor menu. Starting from an initial pixel (termed seed), the algorithm performs a comparison between adjacent pixels (neighbours) and draws closed lines around pixels having the same grey level as the seed. CT images consist in maps of the $\mu(x, y)$ absorption coefficients of the different materials and this allowed to easily implement automatic segmentation tools since the bone tissue offers the greatest contrast with respect to the background material; therefore, the bone structures are the most clearly visible and identifiable also in terms of grey levels [14]. Once all the slides were processed, the related 3D model was generated. The real pelvic surface was digitalized by approximating it to a certain number of polygons (polygonal mesh). In order to reduce the digitalization effects without significant detail loss and to provide a more accurate view, smoothing operations were performed on the 3D model. After the smoothing and filtering operations, the 3D model volume was reduced to less than $1 \%$; therefore, the model geometry was not significantly impacted by the filtering process. The filtered 3D model was compared with the original one, and then 18 fiducial markers were applied on the points of interest of the 3D model. Since the software is able to store the spatial coordinates of each marker, it was possible to evaluate relevant measurement and pelvic angles (Supplement Figure 2).

Patients were categorized according to sex and the following pelvimetry measurements were estimated [12]:

1. Pelvic inlet: obstetric conjugate, true conjugate, and diagonal conjugate (lines from the superior, middle, and inferior pubic symphysis to the sacral promontory); anatomical transverse diameter (farthest distance between iliopectineal lines); and oblique diameter (from the sacroiliac joint to the iliopectineal eminence)

2. Pelvic outlet: straight conjugate (from the lower border of the pubic symphysis to the tip of coccyx); median conjugate (from the lower border of the pubic symphysis to the lower border of the sacrum); bis-ischiatic diameter

3. Other measurements: pubic tubercle height, distance from the sacral promontory to the coccyx; pelvic depth, a line taken from the midpoint of the pelvic inlet to the coccyx; distance from the sacral promontory to S3/S4 
intervertebral disc; distance from S3/S4 intervertebral disc to the tip of the coccyx; and ischial spines distance

4. Angles: pelvic tilt (the angle between the vertical plane and the line that travels through the midpoint of the sacral platform toward the centre of the femoral heads); the offset angle $(\alpha)$, the pelvic inlet angle $(\beta)$, the angle between the inlet, sacral promontory and coccyx $(\chi)$, the angle between the promontory, the coccyx and pelvic outlet $(\delta)$, the angle at $\mathrm{S} 3(\varepsilon)$, and the pelvic inlet to pelvic depth ratio

The bony structure of the pelvis was then 3D modelled to simulate the surgical view when approaching the pelvis transabdominally or trans-perineally. Also, to evaluate how these measures may impact mesorectum distribution, the most representative imaging scans of patients undergoing TaTME procedure for rectal cancer at the Fondazione Policlinico Universitario A. Gemelli in 2019 in Rome were used to provide the surgical perspective concerning mesorectal distribution.

Statistics A priori power analysis computation was performed to estimate the sample size to test the difference between the subgroups using $t$ tests (difference between two independent means) using the following parameters: input: two tails; effect size $d=0.4 ; \alpha$ err prob $=0.05$; power $(1-\beta$ err prob $)=0.80$; allocation ratio $N 2 / N 1=1$. Results obtained were the following: non-centrality parameter $\delta=2.8284271$; critical $t=$ 1.9720175; $\mathrm{Df}=198$, estimating two groups of 100 patients for an actual power of 0.8036475 .

Measurements obtained by pelvimetry calculations were recorded using millimetres and records were de-identified in a database using a consecutive number in each sub-group. All the tests were performed two-tailed and a $p$ value $<0.05$ was considered statistically significant. Statistical analyses were obtained using MedCalc for Windows, version 10.2.0.0, and G*Power software version 3.1.2.

\section{Results}

Study population and pelvimetry Over the study period, 284 patients were considered eligible for the $3 \mathrm{D}$ segmentation based on CT scans (100 patients born in 1955 who underwent imaging in 2015; 66 elective procedures, 30 males and 33 females, and 37 emergency, 28 males and 9 females; 81 patients born in 1956 who underwent imaging in 2016: 58 elective procedures, 29 males and 29 females, and 46 emergency, 28 males and 18 females; 103 patients born in 1957 who underwent imaging in 2017: 57 elective, 29 males and 28 females, and 46 emergency, 28 males and 18 females). The CT scans were reviewed retrospectively according to inclusion/exclusion criteria and revisions stopped when the accrual was achieved to select two groups of 100 female and 100 male patients.

Pelvimetry estimations were performed upon 3D reconstructions and results are presented in Table 1.

Out of 21 pelvimetry measurements, 19 of them documented a significant mean difference between the female and male population. Specifically, pelvic inlet was significantly wider in female vs male patients (Fig. 1). Also, pelvic outlet documented larger diameters for median conjugate and the bisischiatic diameter, with a borderline difference for the straight conjugate. Other measurements and pelvic angles documented dissimilarities of statistical value, with the sole exception of the angle $\varepsilon$ (Figs. 2 and 3).

The narrowness of the pelvis results from the combination of the pelvic inlet, the pelvic depth, and their ratio; in a narrow pelvis, an acute angle of the sacrum $\varepsilon$ could represent almost a perpendicular floor if the dissection is performed from the abdomen, and a vertical one when performed bottom-up (Fig. 3).

Surgical perspective Supplement Video 1 highlights how the bony structure of the pelvis may influence the surgical view when the pelvis is approached from the abdomen or from the perineum in relation to the pelvic inlet, outlet, and angles; in particular, angle $\varepsilon$ could be a hallmark of difficulty when approaching the rectum bottom-up.

Another example of how these measurements may impact the morphology of posterior mesorectum is shown in Fig. 4: remarkably, the posterior/lower mesorectum was very thin in the first patient (narrow pelvis with acute angle $\varepsilon$ ) and well represented in the second one (wider pelvis with obtuse angle $\varepsilon)$. Both these male patients underwent TaTME procedures for colorectal cancers. Indeed, a pelvimetry study may help in defining the burden of dissection, especially when considering the posterior mesorectum (Supplement Video 2).

\section{Discussion}

The recent advantages made in colorectal surgery forced surgeons to change perspective in particular when approaching the anatomy of the rectum "bottom-up". Colorectal surgeons must be aware of the challenges of developing a safe and correct surgical plane in line with the principles of surgical oncology. It is of particular interest the word of cautious used by Philip Quirke commenting on the St. Galle consensus on the safe implementation of TaTME. In his view, "the anatomy is difficult as posteriorly there are complex changes in the angulation of the mesorectal fascial plane and the mesorectum is initially a thin fatty layer. Anteriorly there is very little mesorectum and the anterior surgical margin is in juxtaposition to the urethra". Also, tumour location is a very important factor, since lower tumours can generate worse surgical planes 
Table 1 Pelvimetry measurements in female and male patients

\begin{tabular}{|c|c|c|c|}
\hline & Female patients, $N=100$ & Male patients, $N=100$ & $p$ value \\
\hline \multicolumn{4}{|c|}{ Pelvic inlet } \\
\hline \multicolumn{4}{|c|}{ Obstetric conjugate (mm) } \\
\hline Mean & 126.2 & 119.4 & \\
\hline SD & 8.6 & 9.9 & $4.36544 \mathrm{E}-07$ \\
\hline \multicolumn{4}{|c|}{ True conjugate (mm) } \\
\hline Mean & 122.1 & 114.0 & \\
\hline SD & 8.8 & 10.0 & $4.95965 \mathrm{E}-09$ \\
\hline \multicolumn{4}{|c|}{ Diagonal conjugate (mm) } \\
\hline Mean & 131.8 & 127.9 & \\
\hline $\mathrm{SD}$ & 8.8 & 10.3 & 0.004435735 \\
\hline \multicolumn{4}{|c|}{ Transverse diameter (mm) } \\
\hline Mean & 133.8 & 128.2 & \\
\hline SD & 7.7 & 8.0 & $9.69001 \mathrm{E}-07$ \\
\hline \multicolumn{4}{|c|}{ Oblique diameter (mm) } \\
\hline Mean & 130.0 & 125.7 & \\
\hline $\mathrm{SD}$ & 7.2 & 6.3 & $1.01799 \mathrm{E}-05$ \\
\hline \multicolumn{4}{|c|}{ Pelvic outlet } \\
\hline \multicolumn{4}{|c|}{ Straight conjugate (mm) } \\
\hline Mean & 99.9 & 97.4 & \\
\hline $\mathrm{SD}$ & 10.6 & 8.4 & 0.059169229 \\
\hline \multicolumn{4}{|c|}{ Median conjugate (mm) } \\
\hline Mean & 112.3 & 109.7 & \\
\hline SD & 9.9 & 8.4 & 0.044227539 \\
\hline \multicolumn{4}{|c|}{ Bis-ischiatic diameter (mm) } \\
\hline Mean & 118.0 & 102.2 & \\
\hline $\mathrm{SD}$ & 10.1 & 10.4 & $2.42427 \mathrm{E}-22$ \\
\hline \multicolumn{4}{|c|}{ Other measurements } \\
\hline \multicolumn{4}{|c|}{ Pubic tubercle height (mm) } \\
\hline Mean & 30.7 & 34.8 & \\
\hline SD & 3.7 & 4.0 & $8.51241 \mathrm{E}-13$ \\
\hline \multicolumn{4}{|c|}{ Promontory to coccyx (mm) } \\
\hline Mean & 119.0 & 128.7 & \\
\hline SD & 12.3 & 11.8 & $4.2985 \mathrm{E}-08$ \\
\hline \multicolumn{4}{|c|}{ Pelvic depth (mm) } \\
\hline Mean & 102.8 & 111.5 & \\
\hline SD & 9.8 & 8.8 & $2.6971 \mathrm{E}-10$ \\
\hline \multicolumn{4}{|c|}{ Sacral promontory to S3/S4 (mm) } \\
\hline Mean & 77.2 & 80.6 & \\
\hline SD & 6.7 & 7.8 & 0.001366554 \\
\hline \multicolumn{4}{|c|}{$\mathrm{S} 3 / \mathrm{S} 4$ to coccyx $(\mathrm{mm})$} \\
\hline Mean & 63.4 & 69.6 & \\
\hline SD & 7.7 & 8.6 & $1.90718 \mathrm{E}-07$ \\
\hline \multicolumn{4}{|c|}{ Ischial spines distance (mm) } \\
\hline Mean & 116.7 & 100.5 & \\
\hline $\mathrm{SD}$ & 9.3 & 8.6 & $8.08162 \mathrm{E}-28$ \\
\hline \multicolumn{4}{|l|}{ Angles } \\
\hline \multicolumn{4}{|c|}{ Pelvic tilt (mm) } \\
\hline Mean & 64.7 & 61.0 & \\
\hline SD & 7.7 & 9.0 & 0.001978057 \\
\hline
\end{tabular}


Table 1 (continued)

\begin{tabular}{|c|c|c|c|}
\hline & Female patients, $N=100$ & Male patients, $N=100$ & $p$ value \\
\hline \multicolumn{4}{|c|}{ Offset $\alpha(\mathrm{mm})$} \\
\hline Mean & 32.1 & 27.5 & \\
\hline SD & 3.5 & 3.2 & $9.90042 \mathrm{E}-19$ \\
\hline \multicolumn{4}{|c|}{ Pelvic inlet $\beta(\mathrm{mm})$} \\
\hline Mean & 47.1 & 52.9 & \\
\hline SD & 8.2 & 8.1 & $1.02298 \mathrm{E}-06$ \\
\hline \multicolumn{4}{|c|}{ Angle $\chi(\mathrm{mm})$} \\
\hline Mean & 59.0 & 60.1 & \\
\hline $\mathrm{SD}$ & 7.1 & 5.9 & 0.248535073 \\
\hline \multicolumn{4}{|c|}{ Angle $\delta(\mathrm{mm})$} \\
\hline Mean & 72.7 & 67.6 & \\
\hline SD & 8.9 & 6.9 & $7.32095 \mathrm{E}-06$ \\
\hline \multicolumn{4}{|c|}{ Angle $\varepsilon(\mathrm{mm})$} \\
\hline Mean & 116.4 & 118.5 & \\
\hline SD & 11.8 & 10.0 & 0.17291095 \\
\hline \multicolumn{4}{|c|}{ Pelvic inlet to pelvic depth ratio (mm) } \\
\hline Mean & 1.2 & 1.1 & \\
\hline SD & 0.1 & 0.1 & $8.26754 \mathrm{E}-16$ \\
\hline
\end{tabular}

[15]. The perineal distances and trans-anal angles could be very different in female and male patients. Indeed, the female pelvis tends to be broader, with less prominent ischial spines, whereas in males, the pelvis has usually a longer, more curved sacrum, and a narrower sub-pubic arch, although literature relies on historical publications [16]. In a recent anatomic study, it was documented that the anterior perineum (mean distance between vagina/scrotum and anal verge) is significantly shorter in women comparing men. On the opposite, the rectoperineal angle (measured using a proctoscope) is significantly wider in the female population comparing men (clinical anatomy). If this applies for a proctoscope, it would be consistent to find a difference also when a flexible or rigid trans-anal platform is placed and used in procedures where dissection is based on geometrical tips (the so-called $\mathrm{O}$ and triangle dissection planes) [5] and on a dynamic anatomic distortion made by the pneumorectum [5].

Several authors correlated the anatomical variables with surgical results, for open, robotics, laparoscopic, and TaTME dissections [8, 17-24]. In a small series of patients who underwent open surgery for low rectal tumours, no significant differences were detected between the pelvis depth of females and males. Statistical analyses showed that the body mass index, tumour location, and several pelvimetry parameters, including pelvic inlet, pelvic outlet, height of the pubic symphysis, the sacrococcygeal distance, sacrococcygealpubic angle, and diameter of the upper pubis to the coccyx, significantly affected the operative time, while the tumour's diameter correlated with blood loss. Interestingly, in this study, although patients' clinico-pathological parameters seemed to predict difficulty in low anterior resection, the pelvic anatomical parameters appeared to correlate with variation in abdomino-perineal resection. According to authors' conclusion, the intra-operative difficulty is likely to increase in deeper and narrower pelvises, or in those with greater sacrococcygeal curvature, consistently with the present perspective [20]. Other studies disclosed no significant correlations between pelvic dimensions and operative time in laparoscopic rectal resections, although results were based on a very small cohort of 50 patients [8]. These findings were however not confirmed by others, who documented that a less acutely curved sacrum and a larger sagittal pelvic outlet are significantly correlated with longer operations, especially in a pelvis with a narrow inter-tuberous distance [22]. Consistently, also another larger series of more than 200 patients confirmed that the shape of the pelvic inlet may be useful for predicting the operative time [18]. It should be acknowledged that pelvic bones, although have a key role in determining the shape of the pelvis, may not be sufficient in predicting surgical difficulties and oncologic results: indeed, another study pointed that it is the relative dimensions of the tumour within the pelvis which influence the local recurrence and overall survival rates of patients who underwent open surgery after neo-adjuvant treatment for primary rectal cancer [19]. Another study evaluated the mesorectal fat area calculated at the level of the tip of the ischial spines, in patients undergoing laparoscopic and robotic surgery: according to these researches, a larger mesorectal fat area was associated with 
Fig. 1 Pelvic inlet measurements in female vs male patients. a Obstetric conjugate, two representative patients, and graph bars showing mean difference in the 2 groups. b True conjugate, two representative patients, and graph bars showing mean difference in the 2 groups. c Diagonal conjugate, two representative patients, and graph bars showing mean difference in the 2 groups. $\mathbf{d}$ Transverse diameter, two representative patients, and graph bars showing mean difference in the 2 groups a
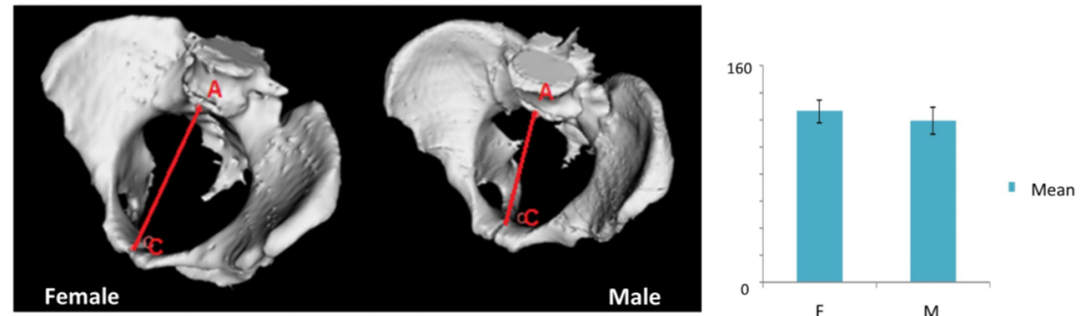

b

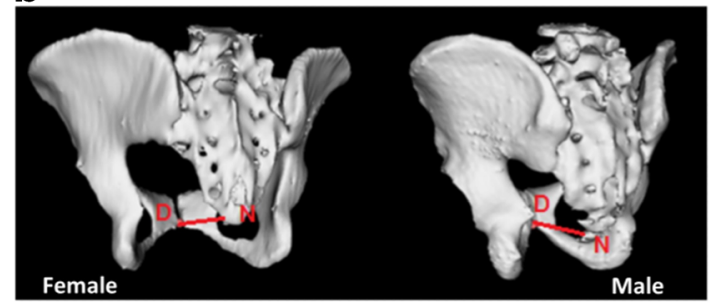

C

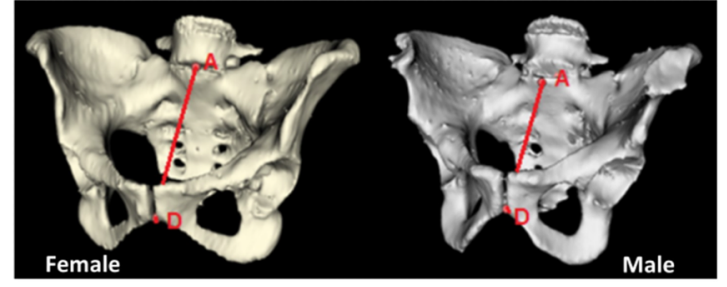

d

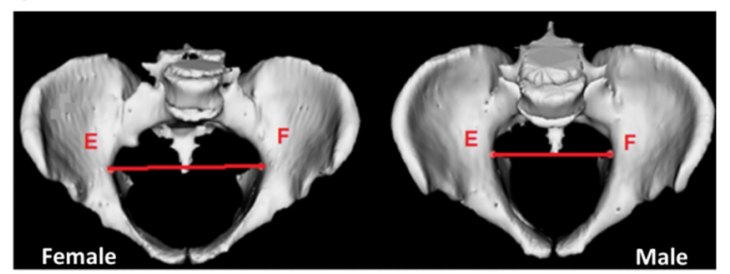

160
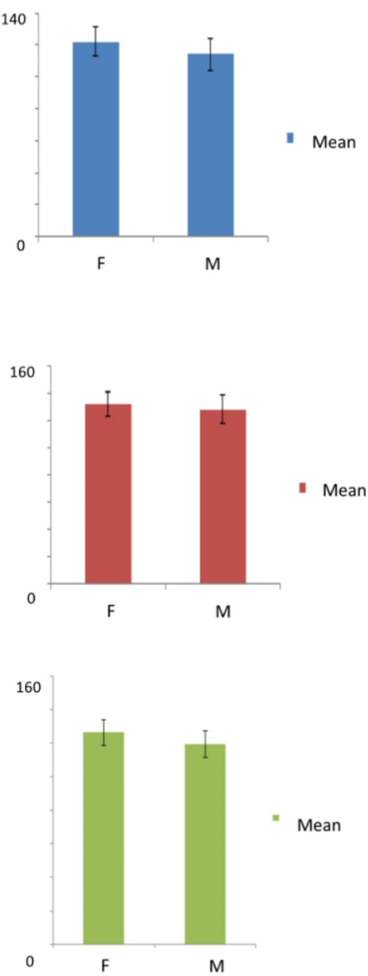

a male patients. a Pelvic tilt, two representative patients, and graph bars showing mean difference in the 2 groups. b Angle $\delta$, two representative patients, and graph bars showing mean difference in the 2 groups
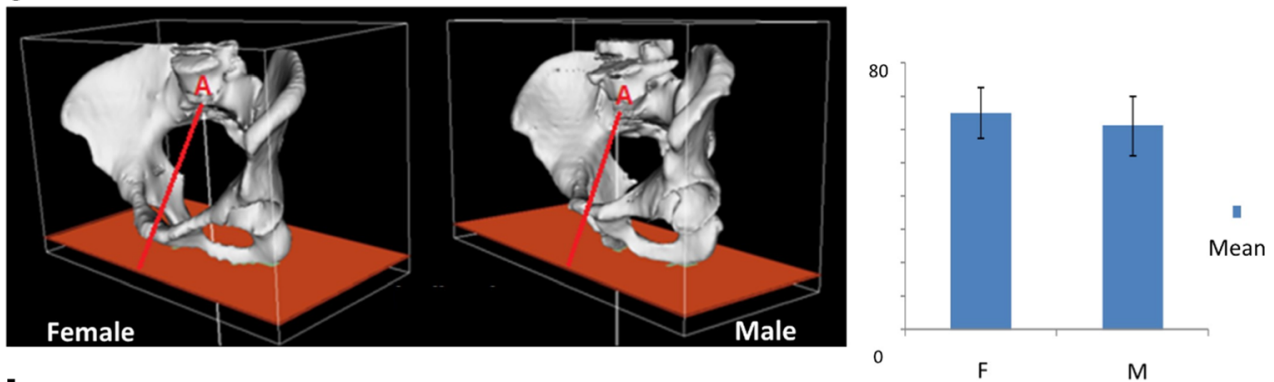

b

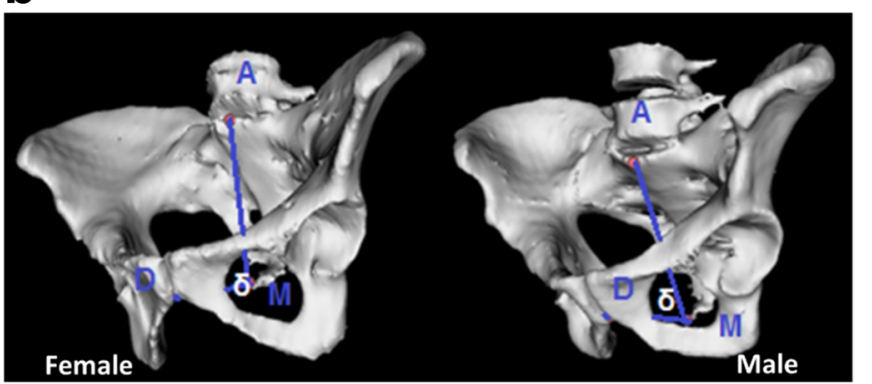

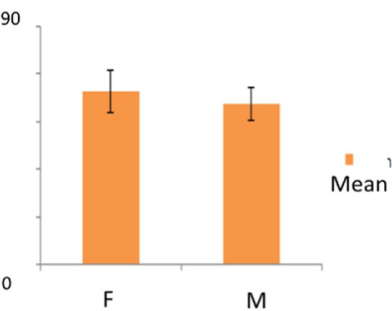


Fig. 3 Pelvic narrowness in female vs male patients. a Angle $\varepsilon$, two representative patients (3D pelvimetry plus vectors representations), and graph bars showing mean difference in the 2 groups. b Pelvic depth, two representative patients, and graph bars showing mean difference in the 2 groups. c Pelvic inlet to pelvic depth ratio, two representative patients, and graph bars showing mean difference in the 2 groups a

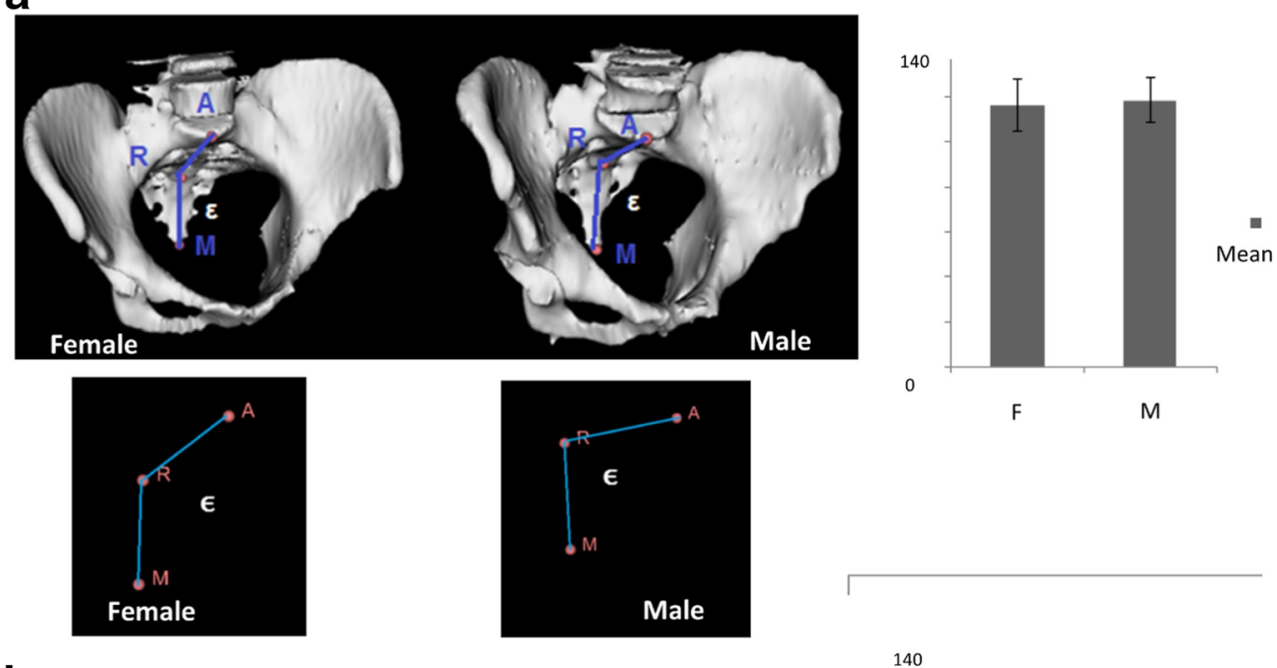

b
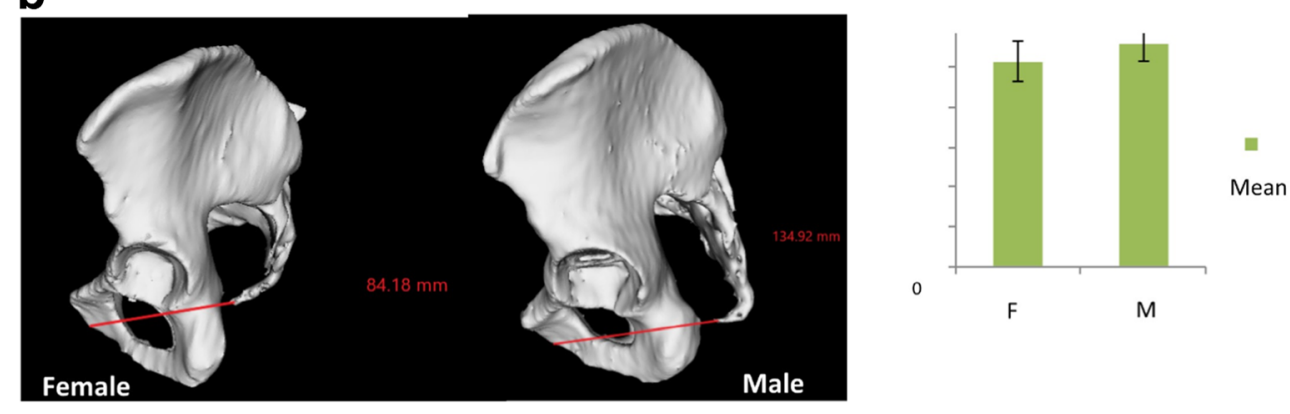

C

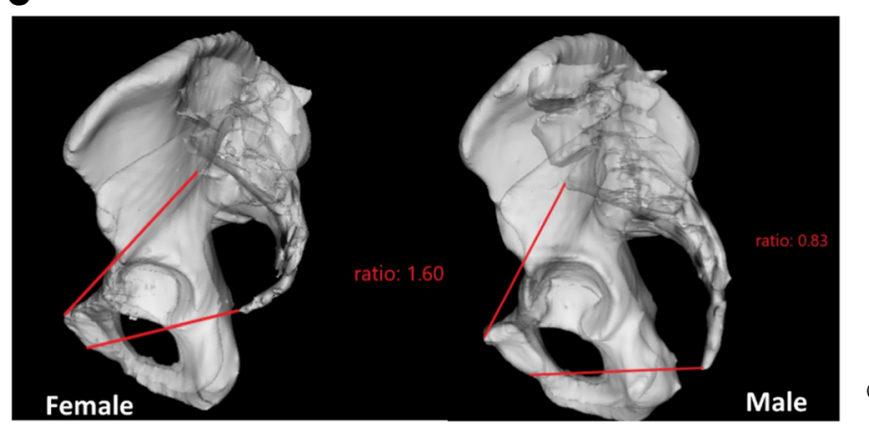

2

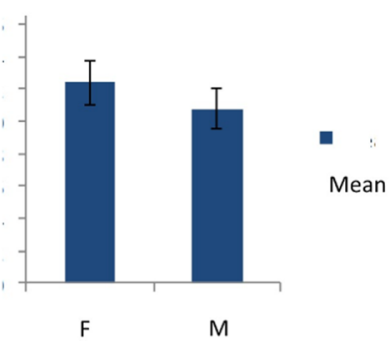

longer operative time of the pelvic phase [20]. More recently, an international collaborative study elaborated a predictive score for operative (time) and post-operative results (hospitalization); this score was calculated based on body mass index (>30), inter-spinous distance $<96.4 \mathrm{~mm}$, ymrT stage $\geq \mathrm{T} 3 \mathrm{~b}$, and male sex, and demonstrated a high accuracy $[23,24]$. Also, in a relatively large cohort of 121 rectal cancer patients undergoing mini-invasive treatment, multivariable analysis indicated that high-grade surgical difficulty was associated with a BMI $>25$, a tumour size $>4.5 \mathrm{~cm}$, an anorectal angle greater than $123^{\circ}$, and pelvic outlet less than $82.7 \mathrm{~mm}$. All of these features were used to develop a scoring model to predict surgical difficulty. The anastomotic leakage rate was $53 \%$ in the high-risk group vs $9.6 \%$ in the low-grade group, and the former had also a significantly higher local recurrence rate comparing the others [25]. This trend was confirmed by a recent meta-analysis that highlighted how bony pelvic measurements may predict surgical difficulty during TME; however, the use of different indicators limits comparison between studies [26].

The bony architecture of pelvis, and in particular the distance between the inter-obturator foramina line and inferior pubic symphysis, the pubic arch angle, pubic ramus width, and pubic symphysis length were investigated also in urologic studies since they may potentially affect surgical procedures such as sub-urethral sling [27]. As far as it concerns specifically nerval injuries in the male population, the study of pelvimetric dimensions did not impact the likelihood of 
Fig. 4 CT and MRI scans in a patient with rectal cancer documenting angle $\varepsilon$ and posterior mesorectum. A1.

Sagittal plane in a male patient with an acute angle $\varepsilon$ represented by vectors on $\mathrm{CT}$ and MRI scans. A2. Same images showing difference when ideally approaching the rectum bottomup. B1. Sagittal plane in a male patient with an obtuse angle $\varepsilon$ represented by vectors on $\mathrm{CT}$ and MRI scans. B2. Same images showing difference when ideally approaching the rectum bottomup. C1. Same patient presented in $\mathrm{A} 1$ and $\mathrm{A} 2$ with acute angle $\varepsilon$, highlighting posterior mesorectum, and $\mathrm{C} 2$ the difference when ideally approaching the rectum bottomup. D1. Same patient presented in $\mathrm{B} 1$ and $\mathrm{B} 2$ with obtuse angle $\varepsilon$, highlighting posterior mesorectum, and D2 the difference when ideally approaching the rectum bottom-up
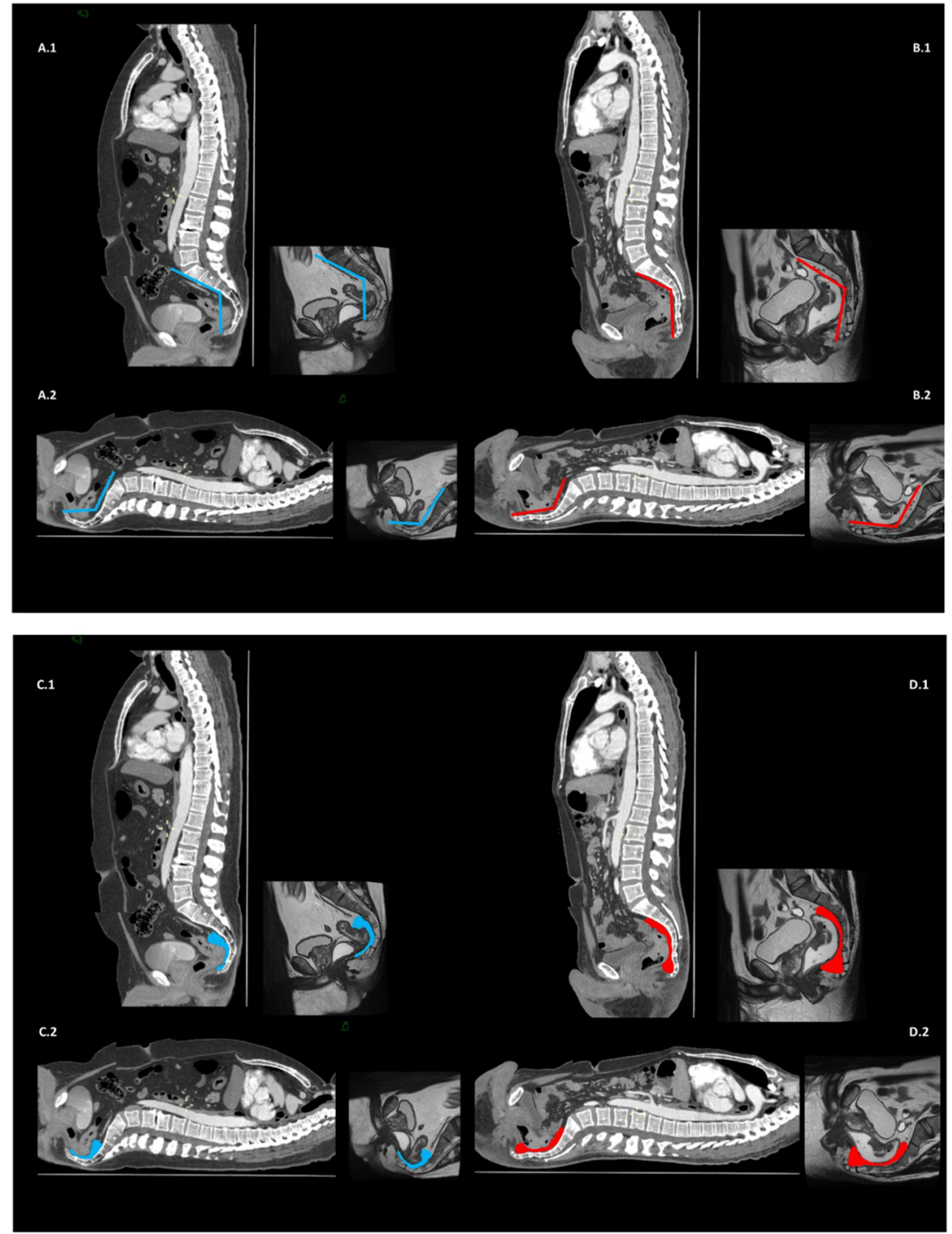

performing a nerve-sparing procedure in patients undergoing radical retropubic prostatectomy [28].

Although these kinds of modelling could be futuristic and not of clinical use for nowadays practice, it is possible that they will be implemented in a short future, in order to help surgeons in the understanding of surgical planes, if required, or implement a trans-abdominal procedure. Consistently with this line of research, a model for the 3D imaging and preoperative planning of a TaTME has been also proposed [17]. Real-time stereotactic navigation for TaTME has been reported to be feasible in small pilot series using laparoscopy, and currently, its feasibility in robotics in combination with indocyanine green fluorescence has been explored to identify structures [29], and the inclusion of 3D pelvimetry may add an additional benefit.

Despite the advantages, TaTME procedures have been also highly criticized for the long-term results reported by some authors, in particular with regard to a higher rate of local recurrence: one example is the Norwegian moratorium by the Norwegian Colorectal Cancer Group [30]. Thus, large international studies are highly advocated. In keeping with this, a large prospective, observational, case-matched, fourcohort, multicentre trial is currently ongoing and designed to study TME using open laparotomy, laparoscopy, robotassisted surgery, or trans-anal surgery in 1300 high-surgicalrisk patients $(\mathrm{BMI}>30$, involvement of the mesorectal fascia upon staging, inter-tuberous distance $<10 \mathrm{~cm}$ ) with mid-tolow, non-metastatic rectal cancer (RESET Study ClinicalTrials.gov Identifier: NCT03574493).

\section{Conclusion}

According to the results of the present study, which investigated a large cohort of patients, differences in the bony structure of the pelvis exist when comparing male and female 
patients, and surgeons must be aware of these differences and of the shape of the pelvis when approaching the rectum, in particular when using a bottom-up technique. Moreover, the perspective highlighted how these differences might have an impact on the distribution of mesorectum, with a consequence on the surgical planning if a total mesoractal excision is required. Increasing the knowledge on pelvis structure and mesorectum distribution may help also in assessing appropriate patients for a trans-abdominal or trans-anal approach.

Supplementary Information The online version contains supplementary material available at https://doi.org/10.1007/s00384-020-03802-9.

Authors' contributions Study conception and design: LL, FB, SQ, EI, FM, and EI. Acquisition of data: LL, FB, FL, SQ, AB, RP, FT, and EI. Analysis and interpretation of data: LL, FB, FL, SQ, GB, FM, AB, RP, DD, FT, and EI. Drafting of the manuscript: LL, GB, FM, RP, DD, and EI. Critical revision of the manuscript: LL, FB, FL, SQ, GB, FM, AB, RP, $\mathrm{DD}, \mathrm{FT}$, and EI.

Funding Open access funding provided by Università Cattolica del Sacro Cuore within the CRUI-CARE Agreement.

\section{Compliance with ethical standards}

Conflict of interest The authors declare that they have no conflict of interest.

Open Access This article is licensed under a Creative Commons Attribution 4.0 International License, which permits use, sharing, adaptation, distribution and reproduction in any medium or format, as long as you give appropriate credit to the original author(s) and the source, provide a link to the Creative Commons licence, and indicate if changes were made. The images or other third party material in this article are included in the article's Creative Commons licence, unless indicated otherwise in a credit line to the material. If material is not included in the article's Creative Commons licence and your intended use is not permitted by statutory regulation or exceeds the permitted use, you will need to obtain permission directly from the copyright holder. To view a copy of this licence, visit http://creativecommons.org/licenses/by/4.0/.

\section{References}

1. Lorenzon L, Bini F, Balducci G, Ferri M, Salvi PF, Marinozzi F (2016) Laparoscopic versus robotic-assisted colectomy and rectal resection: a systematic review and meta-analysis. Int J Color Dis 31: $161-173$

2. Penna M, Hompes R, Arnold S, Wynn G, Austin R, Warusavitarne J, Moran B, Hanna GB, Mortensen NJ, Tekkis PP, TaTME Registry Collaborative (2017) Transanal total mesorectal excision: international registry results of the first 720 cases. Ann Surg 266: $111-117$

3. Persiani R, Biondi A, Pennestrì F, Fico V, De Simone V, Tirelli F, Santullo F, D'Ugo D (2018) Transanal total mesorectal excision vs laparoscopic total mesorectal excision in the treatment of low and middle rectal cancer: a propensity score matching analysis. Dis Colon Rectum 61:809-816
4. Bernardi MP, Bloemendaal AL, Albert M, Whiteford M, Stevenson AR, Hompes R (2016) Transanal total mesorectal excision: dissection tips using 'O's and 'triangles'. Tech Coloproctol 20:775-778

5. Atallah S, Gonzalez P, Chadi S, Hompes R, Knol J (2017) Operative vectors, anatomic distortion, fluid dynamics and the inherent effects of pneumatic insufflation encountered during transanal total mesorectal excision. Tech Coloproctol 21:783-794

6. Barnes TG, Penna M, Hompes R, Cunningham C (2017) Fluorescence to highlight the urethra: a human cadaveric study. Tech Coloproctol 21:439-444

7. Kim NK (2005) Anatomic basis of sharp pelvic dissection for curative resection of rectal cancer. Yonsei Med J 46:737-749

8. Ogiso S, Yamaguchi T, Hata H, Fukuda M, Ikai I, Yamato T, Sakai Y (2011) Evaluation of factors affecting the difficulty of laparoscopic anterior resection for rectal cancer: "narrow pelvis" is not a contraindication. Surg Endosc 25:1907-1912

9. Baik SH, Kim NK, Lee KY, Sohn SK, Cho CH, Kim MJ, Kim H, Shinn RK (2008) Factors influencing pathologic results after total mesorectal excision for rectal cancer: analysis of consecutive 100 cases. Ann Surg Oncol 15:721-728

10. Baek SJ, Kim CH, Cho MS, Bae SU, Hur H, Min BS, Baik SH, Lee KY, Kim NK (2015) Robotic surgery for rectal cancer can overcome difficulties associated with pelvic anatomy. Surg Endosc 29: 1419-1424

11. Zhou X, Su M, Hu K, Su Y, Ye Y, Huang C, Yu Z, Li X, Zhou H, Ni Y, Jiang Y (2015) Applications of computed tomography pelvimetry and clinical-pathological parameters in sphincter preservation of mid-low rectal cancer. Int J Clin Exp Med 8:2174-2181

12. Salerno G, Daniels IR, Brown G, Heald RJ, Moran BJ (2006) Magnetic resonance imaging pelvimetry in 186 patients with rectal cancer confirms an overlap in pelvic size between males and females. Color Dis 8:772-776

13. Lauby-Secretan B, Vilahur N, Bianchini F, Guha N, Straif K, International Agency for Research on Cancer Handbook Working Group (2018) The IARC perspective on colorectal cancer screening. N Engl J Med 378:1734-1740

14. Marinozzi F, Bini F, Marinozzi A, Zuppante F, De Paolis A, Pecci R, Bedini R (2013) Technique for bone volume measurement from human femur head samples by classification of micro-CT image histograms. Ann Ist Super Sanita 3:300-305

15. Adamina M, Buchs NC, Penna M, Hompes R, St. Gallen Colorectal Consensus Expert Group (2018) St. Gallen consensus on safe implementation of transanal total mesorectal excision. Surg Endosc 32:1091-1103

16. Lewis CL, Laudicina NM, Khuu A, Loverro KL (2017) The human pelvis: variation in structure and function during gait. Anat Rec (Hoboken) 300(4):633-642

17. Sahnan K, Pellino G, Adegbola SO, Tozer PJ, Chandrasinghe P, Miskovic D, Hompes R, Warusavitarne J, Lung PFC (2018) Development of a model of three-dimensional imaging for the preoperative planning of TaTME. Tech Coloproctol 22:59-63

18. Shimada T, Tsuruta M, Hasegawa H, Okabayashi K, Ishida T, Asada Y, Suzumura H, Kitagawa Y (2018) Pelvic inlet shape measured by three-dimensional pelvimetry is a predictor of the operative time in the anterior resection of rectal cancer. Surg Today 48: $51-57$

19. Atasoy G, Arslan NC, Elibol FD, Sagol O, Obuz F, Sokmen S (2018) Magnetic resonance-based pelvimetry and tumor volumetry can predict surgical difficulty and oncologic outcome in locally advanced mid-low rectal cancer. Surg Today 48:1040-1051

20. Yamaoka Y, Yamaguchi T, Kinugasa Y, Shiomi A, Kagawa H, Yamakawa Y, Furutani A, Manabe S, Torii K, Koido K, Mori K (2019) Mesorectal fat area as a useful predictor of the difficulty of robotic-assisted laparoscopic total mesorectal excision for rectal cancer. Surg Endosc 33:557-566 
21. Zhou XC, Su M, Hu KQ, Su YF, Ye YH, Huang CQ, Yu ZL, Li XY, Zhou H, Ni YZ, Jiang YI, Lou Z (2016) CT pelvimetry and clinicopathological parameters in evaluation of the technical difficulties in performing open rectal surgery for mid-low rectal cancer. Oncol Lett 11:31-38

22. Killeen T, Banerjee S, Vijay V, Al-Dabbagh Z, Francis D, Warren S (2010) Magnetic resonance (MR) pelvimetry as a predictor of difficulty in laparoscopic operations for rectal cancer. Surg Endosc 24: 2974-2979

23. de' Angelis N, Pigneur F, Martínez-Pérez A, Vitali GC, Landi F, Gómez-Abril SA, Assalino M, Espin E, Ris F, Luciani A, Brunetti F, EuMaRCS Study Group (2019) Assessing surgical difficulty in locally advanced mid-low rectal cancer: the accuracy of two MRIbased predictive scores. Color Dis 21:277-286

24. de Angelis N, Pigneur F, Martínez-Pérez A, Vitali GC, Landi F, Torres-Sánchez T, Rodrigues V, Memeo R, Bianchi G, Brunetti F, Espin E, Ris F, Luciani A, EuMaRCS Study Group (2018) Predictors of surgical outcomes and survival in rectal cancer patients undergoing laparoscopic total mesorectal excision after neoadjuvant chemoradiation therapy: the interest of pelvimetry and restaging magnetic resonance imaging studies. Oncotarget 9: 25315-25331

25. Yamamoto T, Kawada K, Kiyasu Y, Itatani Y, Mizuno R, Hida K, Sakai Y (2020) Prediction of surgical difficulty in minimally invasive surgery for rectal cancer by use of MRI pelvimetry. BJS Open 4:666-677
26. Hong JS, Brown KGM, Waller J, Young CJ, Solomon MJ (2020) The role of MRI pelvimetry in predicting technical difficulty and outcomes of open and minimally invasive total mesorectal excision: a systematic review. Tech Coloproctol 24:991-1000

27. Bogusiewicz M, Rosińska-Bogusiewicz K, Drop A, Rechberger T (2011) Anatomical variation of bony pelvis from the viewpoint of transobturator sling placement for stress urinary incontinence. Int Urogynecol J 22:1005-1009

28. von Bodman C, Matikainen MP, Favaretto RL, Matsushita K, Mulhall JP, Eastham JA, Scardino PT, Akin O, Rabbani F (2011) Pelvimetric dimensions do not impact upon nerve sparing or erectile function recovery in patients undergoing radical retropubic prostatectomy. J Sex Med 8:567-574

29. Atallah S, Parra-Davila E, Melani AGF, Romagnolo LG, Larach SW, Marescaux J (2019) Robotic-assisted stereotactic real-time navigation: initial clinical experience and feasibility for rectal cancer surgery. Tech Coloproctol 23:53-63

30. Larsen SG, Pfeffer F, Kørner H, Norwegian Colorectal Cancer Group (2019) Norwegian moratorium on transanal total mesorectal excision. Br J Surg 106(9):1120-1121

Publisher's note Springer Nature remains neutral with regard to jurisdictional claims in published maps and institutional affiliations. 